\title{
Is Intratympanic Dexamathasone Injection Effective for the Treatment of Idiopathic Sudden Sensorineural Hearing Loss?
}

\author{
Kyu Ho Lee, Sung Hoon Ryu, Hyung Min Lee, Su Kyoung Park, \\ Hyung-Jong Kim, and Jiwon Chang \\ Department of Otolaryngology Head and Neck Surgery, Kangnam Sacred Heart Hospital, Hallym University College of Medicine, \\ Seoul, Korea
}

\author{
Received June 8, 2015 \\ Revised August 18,2015 \\ Accepted September 3, 2015
}

Background and Objectives: The purpose of this study is to identify the effectiveness of intratympanic (IT) steroids when used as an initial therapy in sudden sensorineural hearing loss (SSNHL), and when it is used as a salvage therapy in a refractory SSNHL. Subjects and Methods: This retrospective study included 122 patients with SSNHL. First, patients were divided into three groups according to the initial treatment; IT steroid, systemic steroid and combined (systemic and IT steroid) groups. Second, patients were divided into two groups according to the salvage treatment in a refractory SSNHL; IT steroid and the control (non IT group). Fifty eight patients who did not respond to initial therapy were included in the second analysis. Hearing was assessed immediately before the treatment and 2 weeks, 4 weeks and 3 months after the treatments. Hearing recovery was defined as an improvement of $>15 \mathrm{~dB}$ and the final hearing of $25-45 \mathrm{~dB}$ in the audiogram. Results: When we analyzed the hearing recovery in initial treatment, the comparison of audiogram among three groups did not result in significantly different outcomes. There were no differences in the recovery rate in 2 and 4 weeks throughout all the frequencies. Also, the analysis of the salvage treatment demonstrated that neither IT steroid group nor control group was significantly effective in treating the refractory SSNHL. Conclusions: The results suggest that initial treatment of SSNHL with IT steroid alone is as effective as systemic steroid alone or a combination therapy. Also, salvage IT steroids for refractory SSNHL did not have any additional beneficial effects.

J Audiol Otol 2015;19(3):154-158

KEY WORDS: Sensorineural hearing loss · Intratympanic steroids · Hearing outcome.

\section{Introduction}

Sudden sensorineural hearing loss (SSNHL) is defined as a hearing loss of greater than $30 \mathrm{~dB}$ in at least 3 consecutive frequencies, occurring within 3 days and has a reported incidence of 5 to 20 per 100000 patients every year [1]. There has been a great deal of controversy regarding the possible therapeutic approaches of SSNHL and different treatment modali-

This is an Open Access article distributed under the terms of the Creative Commons Attribution Non-Commercial License (http://creativecommons. org/licenses/by-nc/3.0/) which permits unrestricted non-commercial use, distribution, and reproduction in any medium, provided the original work is properly cited. ties have been tried with no standard protocol universally accepted. At this time, systemic steroids are the only agents with proven effectiveness [2]. The recovery rate in untreated patients (within 3 weeks of onset) ranges from 32 to $65 \%$ in various studies; with systemic steroid therapy, the recovery rate increases to 49 to $89 \%$ [3].

Nevertheless, the systemic administration of steroids is associated with side effects and is contraindicated in certain pathological situations [4]. To address this problem, intratympanic (IT) steroid delivery treatment has gained its popularity for patients who do not respond to standard systemic therapy. IT delivery reduces systemic toxicity and offers significantly high drug levels to the inner ear. Pharmacokinetic animal ex- 
periments have demonstrated that much higher perilymphatic steroid concentrations are achieved with IT applications compared to oral or intravenous administrations [5-7].

IT steroids were first used as salvage therapy in refractory SSNHL. Refractory SSNHL is defined as patients who fail to respond to initial systemic steroid treatment $[8,9]$. Their promising results have made some clinicians promote their use as first-line therapeutic option in patients with contraindication to systemic steroids [10]. Moreover, IT steroid administration has been applied as an adjunctive treatment given concomitantly with systemic steroids. However, the efficacy of steroids in various administration methods remains controversial, and there is no settled definite protocols for IT steroids [11,12]. The aim of this study is to identify the hearing recovery results of IT steroids in the treatment of first occurred SSNHL and refractory SSNHL.

\section{Subjects and Methods}

The records and hearing test results of patients with SSNHL who visited the Department of Otorhinolaryngology in Kangnam Sacred Heart Hospital between January 2012 and April 2014 were analyzed retrospectively. Inclusion criteria for the initial treatment were the following: SSNHL of at least $30 \mathrm{~dB}$ or more at three or more consecutive frequencies, unilateral hearing loss, no history of previous treatment at another clinics, no identified etiologic factors to explain the hearing loss, no history of previous otologic diseases in the affected ear, start for first-line therapy within 30 days after the onset of hearing loss, the ability to complete the treatment protocol of our clinic and patients who were able to attend the clinic for at least a month. Also, the inclusion criteria for the salvage treatment were same as above, but few more things were added; no response or less than $15 \mathrm{~dB}$ improvement in pure tone audiometry (PTA) within 28 days of initial steroid therapy, and the patients who could attend the clinic for at least 3 months.

A total of 122 patients with SSNHL who met the inclusion criteria for initial therapy were selected to participate in the study. First, patients were divided into three groups; IT steroid only, systemic steroid only and combination (systemic and IT steroid) groups. IT steroid (dexamethasone, $5 \mathrm{mg} /$ $\mathrm{mL}$ ) were injected twice a week for 2 weeks [8]. Initial systemic steroids consisted of administration of dexamethasone (10 mg) intravenously for 5 days and followed by oral methylprednisolone $(40 \mathrm{mg}$ ) tapering for 5 days. The combination treatment composed of both IT dexamethasone and administration of intravenous dexamethasone as same as other two groups. IT group consisted of 12 patients, the systemic ste- roids group consisted of 26 patients and combined group 84 patients.

Also, a total of 58 patients were who met the inclusion criteria were selected to participate in the analysis of the effect of the salvage treatment in a refractory SSNHL. Patients were divided into two groups; IT steroid group and the control (non IT group). IT steroid (dexamethasone, $5 \mathrm{mg} / \mathrm{mL}$ ) were injected twice a week for 2 weeks. IT group consisted of 14 patients, and control group 44 patients.

Auditory function was determined by PTA, and mean hearing levels were expressed as the average of hearing thresholds at 500, 1000, 2000, $4000 \mathrm{~Hz}$. To analyze the initial effect of steroids, PTA examinations before the treatment and 14 days, 28 days after the treatment were collected. For the assessment of salvage effect of IT steroids, the PTA on day 28 after start of initial steroid therapy was accepted as the initial audiometric value, and the PTA evaluated at two month after that salvage therapy was used as the final audiometric value.

According to Siegel [13] criteria for hearing improvement, "good recovery" was defined as more than $15 \mathrm{~dB}$ gain and final hearing better than $45 \mathrm{~dB}$, and "poor recovery" was defined as less than $15 \mathrm{~dB}$ gain and final hearing poorer than $45 \mathrm{~dB}$.

\section{Statistical analysis}

All values are expressed as mean $\pm \mathrm{SD}$, and significant differences between groups were determined using Fisher's exact test for categorical variables. For continuous variables, one-way ANOVA was done to identify the differences in three groups and Krusall-Wallis test was conducted to compare between each two groups. $p<0.05$ was defined as the cutoff for statistical significance.

\section{Results}

\section{Initial steroid treatment for SSNHL}

The mean age of the 122 patients included in the study of initial treatment was $52.48 \pm 12.85$ years (range $11-85$ years). Of the participants, $44.3 \%(n=54)$ were men and $55.7 \%$ $(n=68)$ were women. Twelve patients received only IT steroids, 26 patients received systemic steroids, and 84 patients received both IT and systemic steroids for the initial treatment. The descriptive characteristics of the three groups are given in Table 1 . The three groups did not differ significantly except for the interval from onset of symptoms to initiation of therapy. The intervals were $15.83,9.27$, and 7.20 days, respectively, which were statistically significantly different between the groups ( $p=0.004)$.

The initial mean PTA values were $47.50 \pm 12.65 \mathrm{~dB}, 61.65 \pm$ 
Intratympanic Dexamathasone in Idiopathic Sudden Sensorineural Hearing Loss

Table 1. Characteristics of three groups in initial treatment

\begin{tabular}{|c|c|c|c|c|}
\hline & Initial IT dexa & Initial systemic & Initial combined & $p$ value \\
\hline Number & 12 & 26 & 84 & \\
\hline Age $($ mean $\pm S D)$ & $57.33 \pm 17.08$ & $49.96 \pm 12.85$ & $52.57 \pm 12.11$ & 0.259 \\
\hline Gender, n (\%) & & & & 0.250 \\
\hline Male & $7(58.3)$ & $14(53.8)$ & $33(39.3)$ & \\
\hline Female & $5(41.7)$ & $12(46.25)$ & $51(60.7)$ & \\
\hline Ear, n (\%) & & & & 0.737 \\
\hline Right & $7(58.3)$ & $10(38.5)$ & $41(48.8)$ & \\
\hline Left & $5(41.7)$ & $16(61.5)$ & $43(51.2)$ & \\
\hline Occurred days (mean \pm SD) & $15.83 \pm 11.53$ & $9.27 \pm 8.87$ & $7.20 \pm 7.705$ & 0.004 \\
\hline Initial PTA (mean \pm SD) & $47.50 \pm 12.65$ & $61.65 \pm 28.22$ & $62.84 \pm 26.94$ & 0.17 \\
\hline
\end{tabular}

PTA: pure tone audiometry, IT: intratympanic

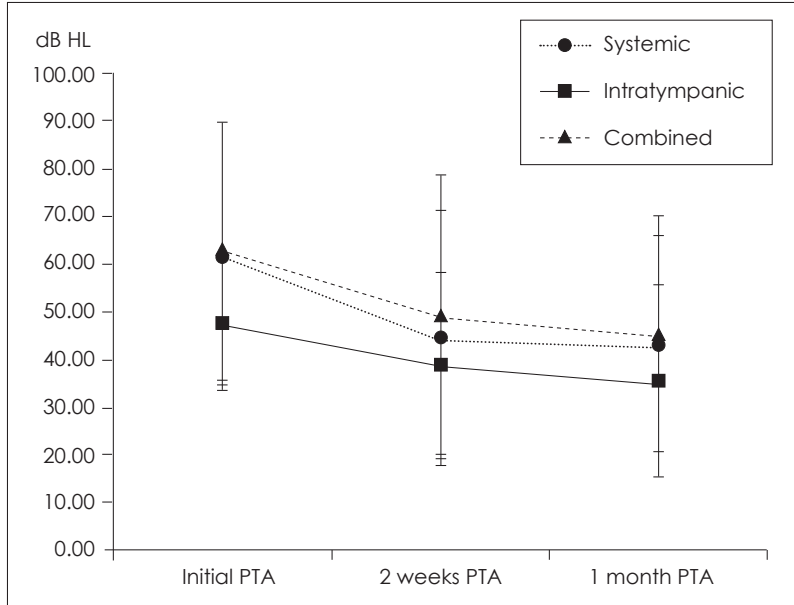

Fig. 1. Hearing thresholds of three groups in initial treatment. The PTA values of patients who received intratympanic steroids were $47.50 \pm 12.65 \mathrm{~dB}$ at first, $39.17 \pm 19.09 \mathrm{~dB}$ in 2 weeks, and $35.56 \pm$ $20.18 \mathrm{~dB}$ in 4 weeks. The PTA values of patients who received systemic steroids were $61.65 \pm 28.22 \mathrm{~dB}$ at first, $44.68 \pm 26.66 \mathrm{~dB}$ in 2 weeks and $43.18 \pm 22.66 \mathrm{~dB}$ in 4 weeks. The PTA values of combination group were $62.84 \pm 26.94$ at first, $49.12 \pm 29.62 \mathrm{~dB}$ in 2 weeks, and $45.51 \pm 24.66 \mathrm{~dB}$ in 4 weeks. The PTA values of each group at same time spot were not different significantly $(p>0.05)$. PTA: pure tone audiometry.

$28.22 \mathrm{~dB}$, and $62.84 \pm 26.94 \mathrm{~dB}$, in the IT, systemic and combined group respectively, and did not differ significantly $(p>0.05)$ (Table 1, Fig. 1). The PTA of patients who received IT steroids improved by $10.78 \pm 16.33 \mathrm{~dB}$, and final PTA after a month was $35.56 \pm 20.18 \mathrm{~dB}$. The PTA of patients who received systemic therapy only improved by $18.23 \pm 17.47 \mathrm{~dB}$, and final PTA after a month was $43.18 \pm 22.66 \mathrm{~dB}$. And the PTA of patients who received both IT and systemic steroids improved by $16.88 \pm 22.15 \mathrm{~dB}$, and final PTA was $45.51 \pm$ $24.66 \mathrm{~dB}$. The degree of hearing recovery and the final PTA were not different significantly among three groups (Fig. 1, 2). Also, hearing recovery rate among three groups did not differ significantly (Table 2).

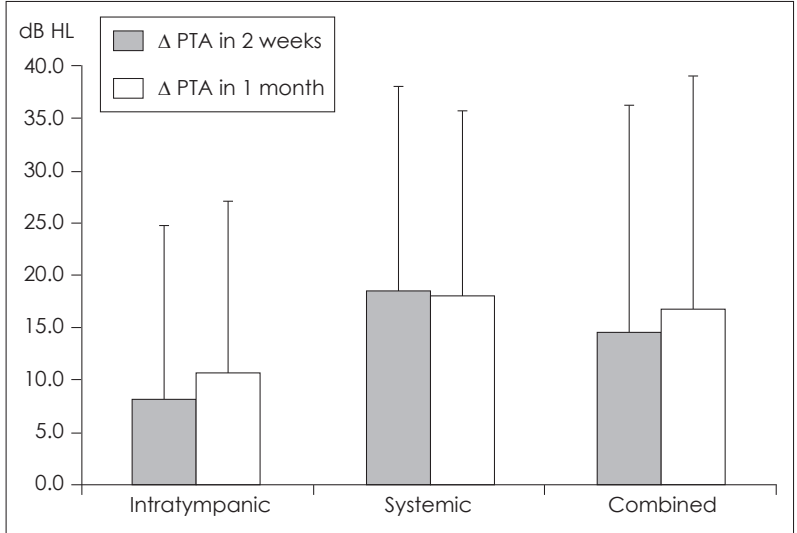

Fig. 2. Hearing gains of three groups in initial treatment. The PTA of patients who received IT steroids improved by $8.33 \pm 16.43 \mathrm{~dB}$ in 2 weeks and $10.78 \pm 16.33 \mathrm{~dB}$ in a month. Patients who received systemic therapy improved by $18.69 \pm 19.36$ in 2 weeks and $18.23 \pm$ $17.47 \mathrm{~dB}$ in a month. Finally, patients who received combined therapy improved by $14.73 \pm 21.48 \mathrm{~dB}$ in 2 weeks and $16.88 \pm$ $22.15 \mathrm{~dB}$ in a month. The degree of hearing recovery and the final PTA were not different significantly $(p>0.05)$. PTA: pure tone audiometry, IT: intratympanic.

\section{Salvage IT steroid treatment for refractory ISSNHL}

The mean age of the 58 patients included in the study of salvage treatment was $54.29 \pm 11.90$ years (range $21-81$ years). Of the participants, $46.6 \%(n=27)$ were men and $53.4 \%$ $(n=31)$ were women. Fourteen patients received salvage intratympanic steroids, and 44 patients did not receive salvage treatment (control). The descriptive characteristics of two groups are given in Table 3. The initial hearing level and the hearing level after a month of initial treatment did not differ significantly in two groups. The audiogram at three month which was the final hearing level after the salvage therapy was $60.44 \pm 17.68 \mathrm{~dB}$ in control group, $59.50 \pm 16.39 \mathrm{~dB}$ in IT group and was not different significantly in both groups (Fig. 3). Also, hearing recovery rate in IT group was $35.7 \%$ and $20.5 \%$ in control group but did not differ significantly (Table 4). 
Table 2. Recovery rate of SSNHL with different initial treatments

\begin{tabular}{|c|c|c|c|c|}
\hline & Initial IT dexa $(n=12)$ & Initial systemic $(n=26)$ & Initial combined $(n=84)$ & p value \\
\hline Recovery rate & & & & 0.476 \\
\hline Good recovery $(n, \%)$ & $7(58.3)$ & $14(53.8)$ & $42(50.0)$ & \\
\hline Poor recovery $(n, \%)$ & $5(41.7)$ & $12(46.2)$ & $42(50.0)$ & \\
\hline
\end{tabular}

SSNHL: sudden sensorineural hearing loss, IT: intratympanic

Table 3. Characteristics of two groups in salvage treatment

\begin{tabular}{lccc}
\hline & IT steroid & Control & p value \\
\hline Numbers & 14 & 44 & \\
Age (mean \pm SD) & $55.36 \pm 8.76$ & $53.95 \pm 12.81$ & 0.647 \\
Gender, $\mathrm{n}(\%)$ & & & 0.063 \\
$\quad$ Male & $10(71.43)$ & $17(38.64)$ & \\
$\quad$ Female & $4(28.57)$ & $27(61.36)$ & \\
Ear, n (\%) & & & 0.543 \\
$\quad$ Right & $5(35.71)$ & $21(47.73)$ & \\
$\quad$ Left & $9(64.29)$ & $23(52.27)$ & \\
Initial PTA (mean \pm SD) & $76.64 \pm 28.5671 .34 \pm 25.15$ & 0.541 \\
1 month PTA (mean \pm SD) & $59.57 \pm 17.4362 .07 \pm 17.43$ & 0.646 \\
\hline
\end{tabular}

PTA: pure tone audiometry, IT: intratympanic

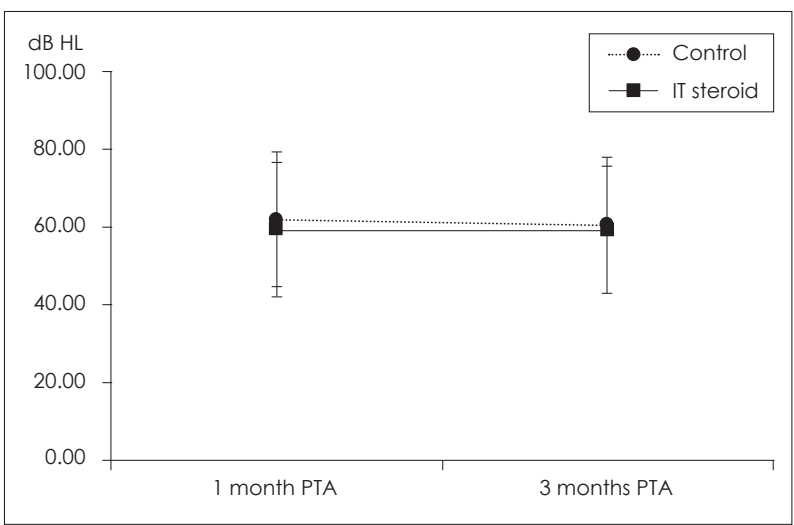

Fig. 3. Hearing thresholds of two groups in salvage treatment. The PTA values of patients who received IT steroids were 59.57士 $17.31 \mathrm{~dB}$ at 1 month (beginning of the salvage treatment), and $59.50 \pm 16.39 \mathrm{~dB}$ at 3 months. The PTA values of control group were $62.07 \pm 17.43 \mathrm{~dB}$ at 1 month and $60.44 \pm 17.68 \mathrm{~dB}$ at 3 months. The finial hearing level after the salvage treatment was not different significantly in both groups $(p>0.05)$. PTA: pure tone audiometry, IT: intratympanic.

\section{Discussion}

There is a continuous controversy regarding the efficacy of IT steroid therapy in ISSNHL. IT steroids are reported to have effect on refractory ISSNHL $[8,9,14]$, and additional IT steroids with systemic therapy also are known to provide effectiveness $[11,12,15]$. However, their reported potency is variable probably due to the different types of steroids used, concentrations, frequencies, injection intervals, and so on.

Ahn, et al. [12] used $5 \mathrm{mg}$ dexamethasone for IT procedure with systemic $48 \mathrm{mg}$ methylprednisolone for initial
Table 4. Recovery rate of ISSNHL with two groups with salvage treatment

\begin{tabular}{lccc}
\hline & $\begin{array}{c}\text { Salvage } \\
\text { IT steroids } \\
(\mathrm{n}=14)\end{array}$ & $\begin{array}{c}\text { Control } \\
(\mathrm{n}=44)\end{array}$ & p value \\
\hline Recovery rate & & & 0.208 \\
Good recovery $(\mathrm{n}, \%)$ & $5(35.7)$ & $9(20.5)$ & \\
Poor recovery $(\mathrm{n}, \%)$ & $9(64.3)$ & $35(79.5)$ & \\
\hline
\end{tabular}

SSNHL: sudden sensorinural hearing loss

combination therapy and reported that combination therapy didn't have additional benefits compared with systemic steroids alone but increased hearing recovery in a lower frequency $(250 \mathrm{~Hz})$. Choi, et al. [16] conducted the investigation with frequent IT dexamethasone administration $(0.3 \mathrm{~mL}$ of 5 $\mathrm{mg} / \mathrm{mL}$ dexamethasone, 5 times per day through the ventilation tube for 5 consecutive days) in addition to intravenous administration of dexamethasone for initial combination therapy and reported that combination therapy didn't have additional benefits compared with systemic steroids. However, Koltsidopoulos, et al. [15] carried out the investigation with 4 mg IT dexamethasone with systemic intravenous prednislone and identified that the combination therapy was effecitvie in mild to severe SSNHL than systemic steroids alone. Our study was conducted with $5 \mathrm{mg}$ IT dexamethasone with intravenous prednisolone, but combination therapy didn't have beneficial effect over systemic steroids. The hearing outcomes achieved by three different protocols that is IT dexamethasone alone, systemic therapy and combination therapy, were not different after 1 month of treatment. However, IT dexamethasone alone had same therapeutic potency with other protocols.

Filipo, et al. [17] proposed that IT treatment as a sole initial treatment. A systematic review assumed that IT steroids can be an important solution for patients with SSNHL who cannot tolerate systemic steroid therapy, such as patients with diabetes [18]. IT steroids are usually administered as either dexamethasone or solumedrol, with wide range of concentrations; most studies on IT corticosteroids refer to dexamethasone 10 to $24 \mathrm{mg} / \mathrm{mL}$ and higher [5]. Although our study was conducted with $5 \mathrm{mg} / \mathrm{mL}$ of dexamethasone, timely injection of steroid had therapeutic effect.

According to the clinical guideline of sudden hearing loss, 
salvage therapy with IT steroid is recommended [8]. Yang, et al. [9] also observed that $5 \mathrm{mg}$ IT dexamethasone induced larger hearing gain (especially at lower frequencies), good recovery rate, and better word recognition score than the control in the treatment of refractory ISSNHL. But in the current study, IT steroid was not effective in the management of refractory SSNHL. IT dexamethasone $(5 \mathrm{mg} / \mathrm{mL})$ was administered within 28 days of start of initial therapy and injected at the anterior superior part of tympanic membrane with a 23 gauge needle, twice a week for 2 week. However, the hearing recovery was not significantly different from the control. Considering the injection protocols were similar to that of initial treatment, the onset of salvage treatment initiation is assumed to be as important as the concentration of the agent.

Our study has some limitations. Because the data were analyzed retrospectively, the subjects could not be randomized to reduce selection bias. In addition, the numbers of patients in each group are not even or large enough to obtain statistically significant differences between the groups. A randomized prospective study with a larger sample is needed to more thoroughly analyze the efficacies IT steroid therapy in the treatment of SSNHL.

\section{Conclusion}

The results of this study suggest that initial treatment of ISSNHL with IT dexamethasone $(5 \mathrm{mg} / \mathrm{mL})$ alone is as effective as systemic steroid or a combination therapy. Also, salvage IT steroids treatment did not have any additional beneficial effects.

\section{REFERENCES}

1) Byl FM Jr. Sudden hearing loss: eight years' experience and suggested prognostic table. Laryngoscope 1984;94(5 Pt 1):647-61.

2) Wilson WR, Byl FM, Laird N. The efficacy of steroids in the treat- ment of idiopathic sudden hearing loss. A double-blind clinical study. Arch Otolaryngol 1980;106:772-6.

3) Moskowitz D, Lee KJ, Smith HW. Steroid use in idiopathic sudden sensorineural hearing loss. Laryngoscope 1984;94(5 Pt 1):664-6.

4) Rauch SD. Intratympanic steroids for sensorineural hearing loss. Otolaryngol Clin North Am 2004;37:1061-74.

5) Parnes LS, Sun AH, Freeman DJ. Corticosteroid pharmacokinetics in the inner ear fluids: an animal study followed by clinical application. Laryngoscope 1999;109(7 Pt 2):1-17.

6) Chandrasekhar SS. Intratympanic dexamethasone for sudden sensorineural hearing loss: clinical and laboratory evaluation. Otol Neurotol 2001;22:18-23.

7) Hargunani CA, Kempton JB, DeGagne JM, Trune DR. Intratympanic injection of dexamethasone: time course of inner ear distribution and conversion to its active form. Otol Neurotol 2006;27:564-69.

8) Stachler RJ, Chandrasekhar SS, Archer SM, Rosenfeld RM, Schwartz SR, Barrs DM, et al. Clinical practice guideline: sudden hearing loss. Otolaryngol Head Neck Surg 2012;146(3 Suppl):S1-35.

9) Yang CH, Wu RW, Hwang CF. Comparison of intratympanic steroid injection, hyperbaric oxygen and combination therapy in refractory sudden sensorineural hearing loss. Otol Neurotol 2013;34:1411-6.

10) Banerjee A, Parnes LS. Intratympanic corticosteroids for sudden idiopathic sensorineural hearing loss. Otol Neurotol 2005;26:878-81.

11) Battaglia A, Burchette R, Cueva R. Combination therapy (intratympanic dexamethasone+high-dose prednisone taper) for the treatment of idiopathic sudden sensorineural hearing loss. Otol Neurotol 2008;29:453-60.

12) Ahn JH, Yoo MH, Yoon TH, Chung JW. Can intratympanic dexamethasone added to systemic steroids improve hearing outcome in patients with sudden deafness? Laryngoscope 2008;118:279-82.

13) Siegel LG. The treatment of idiopathic sudden sensorineural hearing loss. Otolaryngol Clin North Am 1975;8:467-73.

14) Bennett MH, Kertesz T, Perleth M, Yeung P, Lehm JP. Hyperbaric oxygen for idiopathic sudden sensorineural hearing loss and tinnitus. Cochrane Database Syst Rev 2012;10:CD004739.

15) Koltsidopoulos P, Bibas A, Sismanis A, Tzonou A, Seggas I. Intratympanic and systemic steroids for sudden hearing loss. Otol Neurotol 2013;34:771-6.

16) Choi SY, Lee YH, Kim YH. Comparison of the efficacy of systemic and combined highly frequent intratympanic steroid treatment on sudden sensorineural hearing loss. Korean J Audiol 2011;15:133-6.

17) Filipo R, Covelli E, Balsamo G, Attanasio G. Intratympanic prednisolone therapy for sudden sensorineural hearing loss: a new protocol. Acta Otolaryngol 2010;130:1209-13.

18) Seggas I, Koltsidopoulos P, Bibas A, Tzonou A, Sismanis A. Intratympanic steroid therapy for sudden hearing loss: a review of the literature. Otol Neurotol 2011;32:29-35. 